\title{
Evidence for hyperprolactinemia in migraineurs: a systematic review and meta-analysis
}

\author{
Ali Noori-Zadeh ${ }^{1} \cdot$ Morvarid Karamkhani $^{2} \cdot$ Ali Seidkhani-Nahal ${ }^{3}$ (D) $\cdot$ Afra Khosravi $^{4} \cdot$ Shahram Darabi $^{5}$
}

Received: 17 May 2019 / Accepted: 1 August 2019/Published online: 23 August 2019

(C) Fondazione Società Italiana di Neurologia 2019

\begin{abstract}
Background One of the hypothalamus-pituitary axis hormones which may play a crucial role in pathophysiology of migraine is prolactin which is secreted from anterior pituitary gland and synthesized by various immune system cells as well. Whether prolactin blood levels can affect the migraine pathogenesis is an open question. Therefore, investigating prolactin circulatory levels in migraineurs may pave the way to underpin the mechanisms of migraine pathophysiology at biochemical levels. In the current investigation, the prolactin blood levels in the migraine subjects were investigated using systematic review and metaanalysis.

Methods Using online and specialized biomedical databases including Google Scholar, Medline, Pubmed, Pubmed Central, Embase, and Scopus, without the beginning date restriction until Feb 2019, the systematic review retrieved 11 publications in this systematic review after fulfilling for the inclusion and exclusion criteria. For heterogeneity, extent calculation statistical testing was applied. In the present study, the levels of circulatory prolactin in migraineurs assessed using standardized mean difference (SMD) as the effect size.

Results Q quantity and $I^{2} \%$ statistic index showed a high heterogeneity in the 13 selected publications (188.370 and 92.568 , respectively) and random-effects model was chosen for further analyses. The meta-analysis on a total number of 460 migraineurs and 429 healthy controls found that the weighted pooled SMD for the effects of prolactin blood concentrations on migraine pathogenesis was as follows: SMD $=1.435$ (95\% confidence interval, 0.854-2.015).

Conclusion The current investigation presents evidence that prolactin blood levels are higher in migraineurs than healthy subjects.
\end{abstract}

Keywords Prolactin $\cdot$ Migraine $\cdot$ Pain $\cdot$ Pituitary $\cdot$ Aura

\footnotetext{
Article highlights

- Association between blood prolactin and migraine pathogenesis was investigated using meta-analysis.

- The included studies (systematic review) were heterogeneous and random-effects model was applied.

- The pooled SMD for prolactin blood levels was 1.435 (95\% confidence interval, 0.854 - 2.015). $P$ value for the $z$-test was 0.000 .

- Based on the findings, hyperprolactinemia is associated with migraine pathogenesis.
}

Ali Seidkhani-Nahal

Seidkhani-a@medilam.ac.ir; ali_saidkhani@yahoo.com

1 Department of Clinical Biochemistry, Faculty of Allied Medical Sciences, Ilam University of Medical Sciences, Ilam, Iran

2 Department of Epidemiology, Faculty of Health, Ilam University of Medical Sciences, Ilam, Iran
3 Department of Clinical Biochemistry, Faculty of Medicine, Ilam University of Medical Sciences, Ilam, Iran

4 Department of Clinical Immunology, Faculty of Medicine, Ilam University of Medical Sciences, Ilam, Iran

5 Cellular and Molecular Research Center, Qazvin University of Medical Science, Qazvin, Iran 